\title{
Using Hashing to Solve the Dictionary Problem (In External Memory)
}

\author{
John Iacono Mihai Pătraşcu \\ NYU Poly AT\&T Labs
}

November 17, 2021

\begin{abstract}
We consider the dictionary problem in external memory and improve the update time of the wellknown buffer tree by roughly a logarithmic factor. For any $\lambda \geq \max \left\{\lg \lg n, \log _{M / B}(n / B)\right\}$, we can support updates in time $O\left(\frac{\lambda}{B}\right)$ and queries in sublogarithmic time, $O\left(\log _{\lambda} n\right)$. We also present a lower bound in the cell-probe model showing that our data structure is optimal.

In the RAM, hash tables have been used to solve the dictionary problem faster than binary search for more than half a century. By contrast, our data structure is the first to beat the comparison barrier in external memory. Ours is also the first data structure to depart convincingly from the indivisibility paradigm.
\end{abstract}




\section{Introduction}

The case for buffer trees. The dictionary problem asks to maintain a set $S$ of up to $n$ keys from the universe $U$, under insertions, deletions, and (exact) membership queries. The keys may also have associated data (given at insert time), which the queries must retrieve. Many types of hash tables can solve the dictionary problem with constant time per operation, either in expectation or with high probability. These solutions assume a Random Access Machine (RAM) with words of $\Omega(\lg U)$ bits, which are sufficient to store keys and pointers.

In today's computation environment, the external memory model has become an important alternative to the RAM. In this model, it is assumed that there is an memory which is partitioned into pages of $B$ words. Accessing each page in memory takes unit time. The processor is also equipped with a cache of $M$ words ( $M / B$ pages), which is free to access. The model can be applied at various levels, depending on the size of the problem at hand. For instance, it can model the interface between disk and main memory, or between main memory and the CPU's cache.

Hash tables benefit only marginally from the external memory model: in simple hash tables like chaining, the expected time per operation can be decreased to $1+2^{-\Omega(B)}$ [Knu73]. Note that as long as $M$ is smaller than $n$, the query time cannot go significantly below 1 . However, the power of external memory lies in the paradigm of buffering, which permits significantly faster updates. In the most extreme case, if a data structure simply wants to record a fast stream of updates without worrying about queries, it can do so with an amortized complexity of $O(1 / B) \ll 1$ per insertion: accumulate $B$ data items in cache, and write them out at once into a page.

Buffer trees, introduced by Arge [Arg03], are one of the pillars of external memory data structures, along with $B$-trees. Buffer trees allow insertions at a rate close to the ideal $1 / B$, while maintaining reasonably

efficient (but superconstant) queries. For instance, they allow update time $t_{u}=O\left(\frac{\lg n}{B}\right)$ and query time $t_{q}=O(\lg n)$. More generally, they allow the following range of trade-offs:

Theorem 1 (Buffer trees [Arg03]). Buffer trees support updates and queries with the following tradeoffs:

$$
\begin{aligned}
t_{u}=O\left(\frac{\lambda}{B} \lg n\right) & t_{q}=O\left(\log _{\lambda} n\right), & \text { for } 2 \leq \lambda \leq B \\
t_{u}=O\left(\frac{1}{B} \log _{\lambda} n\right) & t_{q}=O(\lambda \lg n), & \text { for } 2 \leq \lambda \leq \frac{M}{B} .
\end{aligned}
$$

In these bounds and the rest of the paper, we make the following reasonable and common assumptions about the parameters: $B \geq \lg n ; M \geq B^{1+\varepsilon}$ (tall cache assumption); $n \geq M^{1+\varepsilon}$.

The motivation for fast (subconstant) updates in the external memory model is quite strong. In applications where massive streams of data arrive at a fast rate, the algorithm may need to operate close to the disk transfer rate in order to keep up. On the other hand, we want reasonably efficient data structuring to later find the proverbial needle in the haystack.

A second motivation comes from the use of data structures in algorithms. Sorting in external memory takes $O\left(\frac{n}{B} \log _{M} n\right)$ which is significantly sublinear for typical values of the parameters. Achieving a bound close to this becomes the holy grail for many algorithmic problems. Towards such an end, a data structure that spends constant time per operation is of little relevance.

Finally, fast updates can translate into fast queries in realistic database scenarios. Database records typically contain many fields, a subset of which are relevant in a typical queries. Thus, we would like to maintain various indexes to help with different query patterns. If updates are efficient, we can maintain more indexes, so it is more likely that we can find a selective index for a future query. (We note that this idea is precisely the premise of the start-up company Tokutek, founded by Michael Bender, Martín Farach-Colton 


\begin{tabular}{c|cc|cc|cc|} 
& \multicolumn{2}{|c|}{$\lambda=B^{\varepsilon}$} & \multicolumn{2}{c|}{$\lg \lg n \leq \log _{M} n$} & \multicolumn{2}{c|}{$M=n^{\varepsilon}$} \\
\hline \multirow{3}{*}{ Buffer Trees } & $t_{u}$ & $t_{u}$ & $t_{q}$ & $t_{u}$ & $t_{q}$ \\
\hline Our Structure & $O\left(1 / B^{1-\varepsilon}\right)$ & $O\left(\log _{B} n\right)$ & $O\left(\frac{1}{B} \log _{M} n\right)$ & $\geq M^{\varepsilon} \lg n$ & $O\left(\frac{\lg n}{B}\right)$ & $O(\lg n)$ \\
$O\left(\frac{\lg \lg n}{B}\right)$ & $2^{\Omega(\lg n / \lg \lg n)}$ \\
& & & & $O\left(\frac{\lg \lg n}{B}\right)$ & $O\left(\frac{\lg n}{\lg \lg \lg n}\right)$
\end{tabular}

Figure 1: Selected update/query tradeoffs for buffer trees and our structure.

and Bradley Kuszmaul. By using buffer-tree technology to support faster insertions in database indexes, the company is reporting 1 significant improvements in industrial database applications.)

The comparison/indivisibility barrier. In internal memory, hash tables can be traced back at least to 1953 [Knu73]. By contrast, in external memory the state-of-the-art data structures (buffer trees, B-trees, many others built upon them) are all comparison based!

The reason for using comparison-based data structures in external memory seems more profound than in internal memory. Consider the simple task of arranging $n$ keys in a desired order (permuting data). The best known algorithm takes time $O\left(\min \left\{n, \frac{n}{B} \log _{M} n\right\}\right)$ : either implement the permutation ignoring the paging, or use external memory sorting. Furthermore it has been known since the seminal paper of Aggarwal and Vitter [AV88] that if the algorithm manipulates data items as indivisible atoms, this bound is tight. It is often conjectured this this lower bound holds for any algorithm, not just those in the indivisible model. This would imply that, whenever $B$ is large enough for the internal-memory $O(n)$ solution to become irrelevant, a task as simple as permuting becomes as hard as comparison-based sort.

While external-memory data structures do not need to be comparison based, they naturally manipulate keys as indivisible objects. This invariably leads to a comparison algorithm: the branching factors that the data structure can achieve are related to the number of items in a page, and such branching factors can be achieved even by simple comparison-based algorithms. For problems such as dictionary, predecessor search, or range reporting, the best known bounds are the bounds of (comparison-based) B-trees or buffer trees, whenever $B$ is large enough (and the external memory solution overtakes the RAM-based solution). It is plausible to conjecture that this is an inherent limitation of external memory data structures (in fact, such a conjecture was put forth by [Yi10]).

Our work presents the first powerful use of hashing to solve the external memory dictionary problem, and the first data structure to depart significantly from the indivisibility paradigm. We obtain:

Theorem 2. For any $\max \left\{\lg \lg n, \log _{M} n\right\} \leq \lambda \leq B$, we can solve the dictionary problem by a Las Vegas data structure with update time $t_{u}=O\left(\frac{\lambda}{B}\right)$ and query time $t_{q}=O\left(\log _{\lambda} n\right)$ with high probability.

At the high end of the trade-off, for $\lambda=B^{\varepsilon}$, we obtain update time $O\left(1 / B^{1-\varepsilon}\right)$ and query time $O\left(\log _{B} n\right)$ (See Figure 11). This is the same as standard buffer trees. Things are more interesting at the low end (fast updates), which is the raison d'être of buffer trees. Comparing to Theorem 11 (1), our results are a logarithmic improvement over buffer trees, which could achieve $t_{u}=O\left(\frac{\lambda}{B} \lg n\right)$ and $t_{q}=O\left(\log _{\lambda} n\right)$.

Interestingly, the update time can be pushed very close to the ideal disk transfer rate of $1 / B$ : we can obtain $t_{u}^{\min }=O\left(\frac{1}{B} \cdot \max \left\{\log _{M} n, \lg \lg n\right\}\right)$.

http://tokutek.com/ 
Note that it is quite natural to restrict the update time to $\Omega\left(\frac{1}{B} \log _{M} n\right)$. Unless one can break the permutation bound, this is an inherent limitation of any data structure that has some target order in which keys should settle after a long enough presence in the data structure (be it the sorted order, or a hash-based order). Since buffer trees work in the indivisible model, they share this limitation. However, the buffer tree pays a significant penalty in query time to achieve $t_{u}=O\left(\frac{1}{B} \log _{M} n\right)$ : from theorem 11 (2), this requires $t_{q} \geq M^{\varepsilon} \lg n$, which is significantly more than polylogarithmic (for interesting ranges of $M$ ). By contrast, our bound on the query time is still (slightly) sublogarithmic.

If one assumes $M$ is fairly large (such as $n^{\varepsilon}$ ), then $\lambda \geq \lg \lg n$ becomes the bottleneck. This is an inherent limitation of our new data structure. In this case, we can achieve $t_{u}=O\left(\frac{\lg \lg n}{B}\right)$ and $t_{q}=$ $O(\lg n / \lg \lg \lg n)$. By contrast, buffer trees naturally achieve $t_{u}=O\left(\frac{\lg n}{B}\right)$ and $t_{q}=O(\lg n)$. With a comparable query time, our data structure gets exponentially closer to the disk transfer rate for updates. If we ask for $t_{u}=O\left(\frac{\lg \lg n}{B}\right)$ in buffer trees, then, from theorem 1 (2), we must have a huge query time of $t_{q}=2^{\Omega(\lg n / \lg \lg n)}$.

Our result suggests exciting possibilities in external memory data structures. It is conceivable that, by abandoning the comparison and indivisibility paradigms, long-standing running times of natural problems such as predecessor search or range reporting can also be improved.

Lower bounds. We complement our data structure with a lower bound that shows its optimality:

Theorem 3. Let $\varepsilon>0$ be an arbitrary constant. Consider a data structure for the membership problem with at most $n$ keys from the universe $[2 n]$, running in the cell-probe model with cells of $O(B \lg n)$ bits and a state (cache) of $M$ bits. Assume $B \geq \lg n$ and $M \leq n^{1-\varepsilon}$. The data structure may be randomized. Let $t_{u}$ be the expected amortized update time, and $t_{q}$ be the query time. The query need only be correct with probability $1-C_{\varepsilon}$, where $C_{\varepsilon}$ is a constant depending on $\varepsilon$.

If $t_{u} \leq 1-\varepsilon$, then $t_{q}=\Omega\left(\lg n / \lg \left(B \cdot t_{u}\right)\right)$.

Remember that for any desired $t_{u} \geq t_{u}^{\min }=\frac{1}{B} \cdot \max \left\{\log _{M} n, \lg \lg n\right\}$, our new data structure obtained a query time of $t_{q}=O\left(\lg n / \lg \left(B \cdot t_{u}\right)\right)$. In other words, we have shown an optimal trade-off for any $t_{u} \geq t_{u}^{\min }$. We conjecture that for $t_{u}=o\left(t_{u}^{\min }\right)$, the query time cannot be polylogarithmic in $n$.

Our lower bound holds for any reasonable cache size, $M \leq n^{1-\varepsilon}$. One may wonder whether a better bound for smaller $M$ is possible (e.g. proving that for $t_{u}=o\left(\frac{1}{B} \log _{B} n\right)$, the query time needs to be superlogarithmic). Unfortunately, proving this may be very difficult. If sorting $n$ keys in external memory were to take time $O(n / B)$, then our data structure will work for any $t_{u} \geq \Omega(\lg \lg n / B)$, regardless of $M$. Thus, a better lower bound for small cache size would imply that sorting requires superlinear time (and, in particular, a superlinear circuit lower bound, which would be a very significant progress in complexity theory).

Remember that update time below $1 / B$ is unattainable, regardless of the query time. Thus, the remaining gap in understanding membership is in the range $t_{u} \in\left[\frac{1}{B}, \frac{\lg \lg n}{B}\right]$.

At the high end of our trade-off, we see a sharp discontinuity between internal memory solutions (hash tables with $\left.t_{u}=t_{q} \approx 1\right)$ and buffer trees. For any $t_{u}<1-\varepsilon$, the query time blows up to $\Omega\left(\log _{B} n\right)$.

The lower bound works in the strongest possible conditions: it holds even for membership and allows Monte Carlo randomization. Note that the error probability can be made an arbitrarily small constant by $O(1)$ parallel constructions of the data structure. However, since we want a clean phase transition between $t_{u}=1-\varepsilon$ and $t_{u}=1$, we mandate a fixed constant bound on the error.

The first cell-probe lower bounds for external-memory membership was by Yi and Zhang [YZ10] in SODA'10. Essentially, they show that if $t_{u} \leq 0.9$, then $t_{q} \geq 1.01$. This bound was significantly strengthened by Verbin and Zhang [VZ10] in STOC'10. They showed that for any $t_{u} \leq 1-\varepsilon$, then $t_{q}=\Omega\left(\log _{B} n\right)$. 
This bound is recovered as the most extreme point on our trade-off curve. However, our proof is significantly simpler than that of Verbin and Zhang. We also note that the technique of [VZ10] does not yield better query bounds for fast updates. A lower bound for small $t_{u}$ is particularly interesting given our new data structure and the regime in which buffer trees are most appealing.

For update time $t_{u} \ll \frac{\lg n}{B}$, our lower bound even beats the best known comparison lower bound. This was shown by Brodal and Fagerberg [BF03] in SODA'03, and states that $t_{q}=\Omega\left(\lg n / \log \left(t_{u} B \lg n\right)\right)$. On

the other hand, in the comparison model, it was possible to show [BF03] that one cannot take $t_{u} \ll \frac{1}{B} \log _{M} n$ (below the permutation barrier) without a significant penalty in query time: $t_{q} \geq n^{\Omega(1)}$.

\section{Upper Bound}

Our data structure is presented in a number of levels. First, in section 2.1, we describe how we can map word-sized keys into keys with $O(\log n)$ bits; a brief discussion of how deletions can be handled using insertions also appears in this preliminary high-level section.

In section 2.2 we then proceed to describe the core component of our structure, called a "gadget." The gadget is defined recursively, and a description of the recursive implementation of the operations is presented in Section 2.2.1 The small non-recursive gadget at the base of the recursion is nontrivial enough to merit a separate description appearing in Section 2.2.2 We present a high-level analysis of the gadget in Section 2.2.3, some probability arguments about the size of gadgets and key-value-collisions are needed, which are isolated in Section 2.2.4

Our gadget has certain size limitations, in that it can only be used as presented if it fits into cache. So, for larger data we use as global recursive structure a variant of buffer trees, where we switch to our gadgets when at levels of the recursion where the size requirements of out gadget are met. We elaborate on this idea in Section 2.3, which completes the description of our structure.

To summarize: after preprocessing ( $\$ 2.1$, a buffer-tree based structure is used, where the leaves are gadgets $(\$ 2.3)$; gadgets are defined recursively $(\$ 2.2)$ with a non-trivial base structure $(\$ 2.2 .2)$.

\subsection{Preliminary key shrinkage and deletions}

As a warm-up, we show how we can assume that keys and associated data have $O(\lg n)$ bits. The data structure can simply log keys in an array, ordered by insertion time. In addition, it hashes keys to the universe $\left[n^{2}\right]$, and inserts each key into a buffer tree with an index into the time-ordered array (of $O(\lg n)$ bits) as associated data. A buffer-tree query may return several items with the same hash value. Using the associated pointers, we can inspect the true value of the key in the logging array. Each takes one memory access, but we know that there are only $O(1)$ false positives with high probability (w.h.p.), with a good enough hash function.

Deletions can be handled in a black-box fashion: add the key to the logging array with a special associated value that indicates a delete, and insert it into the buffer tree normally. Throughout the paper, we will consider buffer trees with an "overwrite" semantics: if the same key is inserted multiple times, with different associated values, only the last one is relevant to the query. Thus, the query will return a pointer to the deletion entry in the log. After $O(n)$ deletions we perform global rebuilding of the data structure to keep the array bounded.

From now on, we assume the keys have $O(\lg n)$ bits. Let $b=\Omega(B \lg n)$ be the number of bits in a page. 


\subsection{Gadgets}

The fundamental building block of our structure is called a gadget. A $t$-gadget stores a multiset $S$ from $([b] \times[t] \times[t]) \times\left[t^{3}\right]$. We refer to the components of a tuple $x=((p, d, s), b) \in S$ as:

- the page hash, $p \in[b]$;

- the distribution hash, $d \in[t]$;

- the shadow hash, $s \in[t]$;

- the backpointer, $b \in\left[t^{3}\right]$. Taken together, the page, distribution, and shadow hash codes are treated as a key, with the backpointer being associated data.

Operations. A $t$-gadget stores a multiset supporting two operations:

BULK-INSERT $(T)$ : Insert a multiset $T \subseteq\left([b] \times[t]^{2}\right) \times\left[t^{3}\right]$ into the data structure $(S \leftarrow S \cup T)$. The multiset is presented packed into $O\left(\left\lceil|T| / \frac{b}{\lg t}\right\rceil\right)$ pages.

QUERY $(x)$ : Given a key $x \in[b] \times[t]^{2}$, return the (possibly empty) list of the backpointer values of all elements of $S$ with key value $x$. We aim for time bounds proportional to the number of occurrences of elements with key value $x$ in the multiset.

Capacity invariants. Our construction will guarantee that the the number of keys stored in a $t$-gadget is $|S|=O(b t)$ with high probability. A $t$-gadget will occupy $O\left(|S| / \frac{b}{\lg t}\right)=O(t \lg t)$ pages thanks to the use of a succinct encoding. At the beginning of any operation, there is no guarantee that any part of the $t$-gadget is in cache. However, we will only use gadgets that can completely fit in cache, i.e. the entire gadget can be loaded in cache by the update algorithm if desired.

A recursive construction. At a high level, our data structure follows the same recursive construction used in the van Emde Boas layouttwo types of $t$-gadgets: the recursive t-gadget, and the base t-gadget which is used as a base case for small $t$. The description of the base $t$-gadget is deferred to 2.2.2. Here we define the recursive $t$-gadget.

Recursive $t$-gadgets contain the following components:

- The log: An array containing all the elements of $S$ in the order of insertion. The last block of the array is the only one which may be partially full and is referred to as the tail block of the log.

- The top gadget $g^{T}$ : A a recursive $\sqrt{t}$-gadget.

- The bottom gadgets $g_{i}^{B}$ : An array of $\sqrt{t} \sqrt{t}$-gadgets.

All elements of $S$ are stored in the log; furthermore, all elements of $S$ except for those in the tail block will have a truncated representation of their keys recursively stored in either the top gadget or one of the bottom gadgets. Formally:

Invariant 4. Let $\mathrm{HIGH}(\cdot)$ and $\mathrm{LOW}(\cdot)$ refer to the most and least significant half of the bits of their parameter. Given an element $x=((p, d, s), b)$ exactly one of the following holds:

1. Element $x$ is stored in the tail block of the log.

2. Element $x$ is stored in block $i$ of the $\log$ and $((p, \operatorname{HIGH}(d), \operatorname{HIGH}(s)), i)$ is stored in the top gadget, $g^{T}$. The element $((p, \operatorname{HIGH}(d), \operatorname{HIGH}(s)), i)$ is called the "top-compressed" key. 
3. Element $x$ is stored in block $i$ of the $\log$ and $((p, \operatorname{LOW}(d), \operatorname{LOW}(s)), i)$ is stored in $g_{\mathrm{HIGH}(d)}^{B}$. The element $((p, \operatorname{LOW}(d), \operatorname{LOW}(s)), i)$ is called the "bottom-compressed" key.

Given an element $x$ stored in a $t$-gadget, the top-compressed version of $x$ consists of the entire page hash of $x$, and the $\frac{1}{2} \lg t$ higher-order bits of the distribution hash and shadow hash. The bottom-compressed version of $x$ is constructed analogously using the page hash and and lower-order bits of the distribution hash and shadow hash. In both cases the backpointer indicates the page containing $x$ in the log of the $t$ gadget. These compressed elements meet the size requirements for elements that can be bulk-inserted into $\sqrt{t}$-gadget; i.e. the compressed key by definition is an element of $[b] \times[\sqrt{t}]^{2}$. Since $|S|=\Theta(b t)$, there are at most $\Theta(t \lg t)$ pages in the $\log$, so the backpointer is easily within the required $\left[t^{3}\right]$.

The backpointer serves the following purpose: given a top or bottom compression of some element $x$ relative to a specific and known $t$-gadget, $x$ can be determined in $O(1)$ time by simply using the backpointer of the compression as an index into the $\log$ of the $t$-gadget. In this way the bits removed from the distribution and shadow hashes of of $x$ to form its compression can be restored; as we will see, this allows the support of "uncompression" as recursive queries return.

\subsubsection{Implementation of gadget operations}

Query. A QUeRY $(x)$ operation proceeds as follows, where $x=(p, d, s)$ is the current recursive compression of the original hashed key:

- Inspect the tail block of the log, and retrieve the associated backpointers of all occurrences of $x$ from there. This takes one block read, and returns all the backpointers of the data satisfying case 1 of invariant 4

- Recursively call QUERY in the top gadget $g^{T}$ with the top-compressed key $(p, \operatorname{HIGH}(d), \operatorname{HIGH}(s))$. The top gadget will return a set of backpointers $\left\{p_{1}, p_{2}, \ldots\right\}$, which are indexes into the $\log$ of the current gadget. The set of items from the log includes all data satisfying case 2 of invariant 4 , plus possibly some false positives, where the compressed key matches the query key from the perspective of the top gadget, but not the full key is different. For any result returned by the top gadget, the query inspects the key in the log (taking constant time for the pointer access) and verifies the lower halves of the distribution and shadow hash codes, $\operatorname{LOW}(d)$ and LOW $(s)$. If any of these differ, the result is a false positive and is discarded. Otherwise, the result is returned along with the original backpointer, retrieved from the log. We will later need to bound the number of false positives induced by hashing and compression.

- Recursively call QUERY in the bottom gadget $g_{\mathrm{HIGH}(d)}^{B}$ with the compressed key ( $p$, LOW $(d)$, LOW $\left.(s)\right)$. This returns all data satisfying case 3 of Invariant 4 together with some false positives that may be introduced by trimming the shadow hash code. For every recursive result, the query accesses the appropriate page in the log through the returned backpointer, and verifies that the lower half of the shadow hash code LOW $(s)$ matches the key. If not, the result is discarded as a false positive. In case of a match, the original backpointer from the log is returned to the parent.

Observe that recursing in the bottom gadget cannot introduce a false positive due to the distribution hash, since it is only keys with the same top bits as $x$ that appear in the gadget.

Bulk-Insert. An insertion proceeds as follows:

1. Add the inserted items at the end of the log. 
2. If one or more than one blocks are filled as a result of step 1, the recursive top compressed representation of the data in the newly filled blocks is computed and the resultant top-compressed data is bulk-inserted into the top gadget. We call this a little flush.

3. If the top gadget contains $b \sqrt{t}$ keys as a result of (2), it is declared to be full. It is then "destroyed" (initialized to an empty state) and all keys previously stored in the top gadget are bottom-compressed and inserted into the appropriate bottom gadget. We call this a big flush. To efficiently implement this operation, the data to be flushed is copied from the log, where it appears contiguously in uncompressed form, into cache, where it all fits according to the capacity invariant. There the data is bucketed (for free using any sorting algorithm, since we are in internal memory) into $\sqrt{t}$ groups depending on the $\operatorname{HIGH}(d)$ field which indicates which recursive gadget it should be inserted into. Once the bucketing is complete, the data is converted into the appropriate compressed form, $\sqrt{t}$ recursive BULK-INSERT operations are executed in the bottom gadgets.

Note that this procedure enforces Invariant 4 , by ensuring each item is either in the tail block of the log, in the top gadget, or in a bottom gadget. The capacity requirement of the top gadget is explicitly enforced. The capacity requirement of any bottom gadget holds w.h.p; this is argued in Section 2.2.4.

\subsubsection{Base case}

We switch to the base case of the recursion when $t \leq t^{\mathrm{min}}$, for a parameter $t^{\mathrm{min}}$ to be determined (see $\$ 2.3$ ). To achieve the full range of our trade-offs, we need to use a different non-recursive construction for these small gadgets. Such a gadget maintains a single buffer page with the last $\leq \frac{b}{\lg t}$ inserted keys. The rest of the keys are simply stored in a hash table (e.g. collision chaining) addressed by the page hash. This is the one place where the page hash is used. With a succinct representation, the table occupies $O\left(|S| / \frac{b}{\lg t}\right)=O(t \lg t)$ pages.

QUERY $(x)$ inspects the buffer page and only one page of the hash table w.h.p. (since we have assumed $B=\Omega(\lg n)$, and the maximal chain is $O(\lg n / \lg \lg n)$ w.h.p.). Thus, a query takes time $O(1)$. UpDATE simply appends keys to the buffer page. When the buffer page fills, all keys are inserted into the hash table. This operation may need to touch all $O(t \lg t)$ pages, since the new keys are likely to have hash codes that are spread out; however the cost can not exceed $O(t \lg t)$ since the capacity invariants ensure the whole gadget fits in memory.

\subsubsection{Analysis of the $t$-gagdet}

In this section, we analyze the performance of gadgets, delaying the probabilistic analysis to the next section.

Space usage. Though a key stored in a gadget may appear in $O(\lg \lg n)$ recursive gadgets, the repeated compression results in the space used by all compressed occurrences of the key is dominated by the toplevel representation of the key. Formally, a single key in a $t$ gadget occupies $O(\lg t)$ bits of space and may appear recursively in at most one $\sqrt{t}$-gadget. Thus, the space per key is given by the recurrence $S(t) \leq S(\sqrt{t})+O(\lg t)$, which solves to $O(\lg t)$ bits. Overall, a $t$-gadget storing $n$ keys uses $O(n)$ words of space.

Update cost. Over its lifetime in a $t$-gadget, an element will be appended to the log once, participate in a little flush (being inserted into the top gadget) at most once, and participate in a a big flush (being moved into a bottom gadget) at most once. 
We begin our analysis with the cost of BULK-INSERT, excluding recursive calls. A key participates in a BULK-INSERT operation if it is one of the inserted items or participates in a big or little flush. If $k$ is the number of participating items, the actual running time excluding recursive calls, is $O\left(1+\frac{k \lg t}{b}\right)$. Since $\frac{k \lg t}{b}$ is the (fractional) number of blocks occupied by a single element in a $t$-gadget, this is the fastest possible (linear time). Let us briefly explain how this is achieved for each of the three steps presented in the description of the BULK-INSERT operation. The first step, inserting at the end of the buffer, can be done efficiently since we required that the inserted data is already presented packed into pages. In the little flush, one or more blocks of data from the log need to be copied and converted into top-compressed form and delivered to single recursive BULK-INSERT; this can be done with a simple scan. The big flush, by definition is performed when the top $\sqrt{t}$-buffer is full, containing $b \sqrt{t}$ keys; the actual cost (including the calling of but excluding the execution of the recursive bulk insertions) is $\Theta(\sqrt{t} \log \sqrt{t}+\sqrt{t})$, with the $\sqrt{t}$ being a lower-order term due to the $\sqrt{t}$ recursive calls to BULK-INSERT.

We now bound the recursive cost by amortizing. To cover the constant additive term, we assign an amortized $O(1)$ credit to every BULK-INSERT operation. The credit for the recursive call in the top gadget can be paid because a recursive call is only made when we fill a page of the log. The credit for the recursive calls in the bottom gadgets is a lower order term compared to sorting the entire top gadget.

Now we are left with a cost of $O\left(\frac{k \lg t}{b}\right)$ for a BULK-INSERT operation in which $k$ keys participate. This translates into an amortized cost of $O\left(\frac{\lg t}{b}\right)$ per key. Let $U(t)$ be the total cost charged to a key by a $t$-gadget, including recursive calls. This is described by the recurrence:

$$
U(t)=O\left(\frac{\lg t}{b}\right)+2 \cdot U(\sqrt{t}) ; \quad U\left(t \leq t^{\min }\right)=O\left(\frac{t \lg t}{b}\right)
$$

Observe that on each level of the recurrence, we have $2^{i}$ terms of $O\left(\frac{1}{b} \lg \left(t^{-2^{i}}\right)\right)$, i.e. a constant total cost of $O\left(\frac{\lg t}{b}\right)$ per level. This is very intuitive: at each level, our key is broken into many components, but their total size stays $\lg t$ bits. Since the cost is proportional to the bit complexity of the key, the cost of each level is constant; e.g. at the top level you recurse twice on keys of half the size. This property of the data structure is the most crucial element in obtaining our upper bound. It only possible due to our compression of the keys; without compression, i.e. without violating indivisibly, the cost would increase geometrically at each level instead of remaining unchanged.

The recursion for $U(t)$ solves as follows: the recursion has $O(\lg \lg t)$ levels at a cost of $O\left(\frac{\lg t}{b}\right)$ per level. In the base case, the recursion has $\lg t / \lg t^{\mathrm{min}}$ leaves, each of cost $O\left(\frac{1}{b} t^{\min } \lg t^{\mathrm{min}}\right)$. Thus the total cost is $U(t)=\frac{\lg t}{b} \cdot O\left(\lg \lg t+t^{\min }\right)$.

Query cost. The query time is proportional to the number of (true positive) results returned. Let $Q(t)$ be the cost per result of a query in a $t$-gadget. We first note that $Q(t)$ is at least 1 , because any result has to be looked up in the log, in order to check whether it is a true positive. The query cost is proportional to the number of gadgets traversed, and is described by the easy recursion:

$$
Q(t)=1+2 \cdot Q(\sqrt{t}) ; \quad Q\left(t^{\min }\right)=1
$$

The number of gadgets grows exponentially with the level, and is dominated by the base case. The total cost is therefore $Q(t)=O\left(\lg t / \lg t^{\mathrm{min}}\right)$.

For each false positive encountered throughout the query, there is an additional cost of at most $O\left(\lg \frac{\lg t}{\lg t^{\min }}\right)$. Indeed, each key is in at most $O\left(\lg \frac{\lg t}{\lg t^{\min }}\right)$ levels at a time, and we need to do $O(1)$ work per level: we go to the appropriate page in the log to verify the identity of the key and retrieve its data. We will show later that the total overhead due to false positives is $O(Q(t))$ w.h.p. 


\subsubsection{Probabilistic Analysis}

Capacity bounds. We first prove that no $t$-gadget $g$ receives more than $O(b t)$ keys w.h.p. Note that shadow and page hashes are irrelevant to this question, and we only need to analyze distribution hashes. For now, assume that our hashing is truly random.

Let $g^{\prime}$ be the lowest ancestor of $g$ in the recursion tree which is a top-recursive gadget of its parent; say $g^{\prime}$ is a $t^{\prime}$-gadget. We conventionally interpret the root gadget to be a top-recursive gadget, so $g^{\prime}$ is always defined. Note that $g^{\prime}$ could be $g$. Remember that BULK-INSERT enforces a worst-case capacity bound on any top gadget by the big flush operation, so the number of keys of $t^{\prime}$ is at most $b \cdot t^{\prime}$ in the worst case. A key from a $t^{\prime}$-gadget ends up in a specific bottom gadget only if the first half of its distribution hash matches the identity of the bottom gadget. Recursively, keys that end up in a specific grandchild $\sqrt[4]{t}$-gadget have the same prefix of $\frac{3}{4} \lg \left(t^{\prime}\right)$ bits of the distribution hash. Since $g^{\prime}$ is the lowest ancestor of $g$ that is a top-recursive gadget, all keys of $g^{\prime}$ that end up in $g$ do so through bottom recursion, i.e. they will all have a common prefix of $\lg \left(t^{\prime}\right)-\lg t$ bits. Therefore, analyzing the number of keys of $g^{\prime}$ that end up in $g$ is a standard balls-in-bins problem with the $b t^{\prime}$ balls of $g^{\prime}$ being distributed uniformly into $\frac{t^{\prime}}{t}$ bins. The expected number of balls landing in gadget $g$ is $t b$. Since $b=\omega(\lg n)$, the Chernoff bound says that we have at most $O(t b)$ keys in the bin with high probability in $n$.

False positives during QUERY. We now switch to analyzing the number of false positives encountered by QUERY(x). We count a false positive only once, in the first level of recursion where it is introduced. As noted above, a false positive introduced in a $t$-gadget induces an additive cost of $O\left(\lg \frac{\lg t}{\lg t^{\min }}\right)$ on the query time.

We claim that for any $t$, the number of the false positives introduced in all $t$-gadgets that the query traverses is $O\left(\log _{t} n\right)$ w.h.p. Thus, the total cost on one level of the recursion is $O\left(\frac{\lg n}{\lg t} \cdot \lg \frac{\lg t}{\lg t^{\min }}\right)$. At the $i$-th level of the recursion, we have $\lg t=2^{i} \lg t^{\mathrm{min}}$, so the total cost is:

$$
\sum_{i} O\left(\frac{\lg n}{\lg \left(2^{i} \lg t_{\text {min }}\right)} \cdot \lg \frac{\lg \left(2^{i} \lg t^{\text {min }}\right)}{\lg t^{\text {min }}}\right)=O\left(\frac{\lg n}{\lg t^{\text {min }}}\right) \sum i 2^{-i}=O\left(\frac{\lg n}{\lg t^{\text {min }}}\right)=O(Q(t)) .
$$

Thus, the total cost due to false positives is $O(Q(t))$ w.h.p.

We must now prove our claim that the false positives introduced in all $t$-gadgets on the query path is $O\left(\log _{t} n\right)$ w.h.p. There are $\log _{t} n t$-gadgets on the path of the query, and each cares about a disjoint interval of $\lg t$ bits of the distribution and shadow hash codes. For the analysis, we imagine fixing a growing prefix of the distribution and shadow hashes, in increments of $\lg t$ bits. At every step, the fixing so far decides which keys land in the next $t$-gadget. Among these, only the most recent $\sqrt{t} \cdot b$ can be in the top gadget at query time. One of these keys is a false positive iff it is different from the query, yet its page hash and the top half of its distribution and shadow hash codes match the query key. These hash codes consist of $\lg b+2 \lg \sqrt{t}$ random bits, so this event happens with probability $\frac{1}{b t}$ independently for each of the $\sqrt{t} \cdot b$ keys. For bottom compression, a key can only be a false positive iff it is different from the query, yet its page hash, full distribution hash, and the bottom half of the shadow hash match the query. These hash codes have $\lg b+\frac{3}{2} \lg \sqrt{t}$ random bits together, so a key is a false positive in a bottom recursion with probability $\frac{1}{b t^{3 / 2}}$. Over the path of the query through $\log _{t} n$ different $t$-gadgets, there are $b \sqrt{t} \cdot \log _{t} n$ keys that could become false positives through top recursion (each independently with probability $\frac{1}{b t}$ ), and w.h.p. $b t \cdot \log _{t} n$ keys that could become false positive through bottom recursion (each independently with probability $\frac{1}{b t^{3 / 2}}$ ). The expected number of false positives among all $t$-gadgets is therefore $O\left(\frac{\log _{t} n}{\sqrt{t}}\right)$. The Chernoff bound (in the 
Poisson-type regime) shows that the number of false positives does not exceed $O\left(\log _{t} n\right)$ with probability $(1 / \sqrt{t})^{\Omega\left(\log _{t} n\right)}=2^{-\Omega(\lg n)}$, i.e. with high probability in $n$.

Since our analysis only relies on Chernoff bounds, it holds even with weaker hash functions that satisfy such bounds. The main requirement for the hash function is that it satisfy Chernoff-type concentration even among the set of keys that share a certain prefix of the hash code. This is true of $k$-independent hash functions, since a $k$-independent distribution remains $k$-independent if we fix a prefix of the bits of the outcome. Thus, a $\Theta(\lg n)$-independent hash function suffices for our data structure, considering Chernoff bounds with limited independence given by [SSS95]. These hash functions can be represented in $O(\lg n)$ words, which fits in cache, and therefore can be evaluated without I/O cost.

\subsection{Putting Everything Together}

We now describe how gadgets are used to make a dictionary structure that supports INSERT and SEARCH operations. Recall that the capacity invariant of our gadget requires that that the total size of a gadget is smaller than the cache size, so a single gadget in and of itself cannot be the target data structure.

Our data structure is globally organized like the original buffer tree, except that each node is implemented by a gadget to support fast queries. At the global level, only comparisons are needed.

Formally, we have a tree with branching factor $M^{\varepsilon}$. Each node can store up to $M$ keys. These keys are stored in a plain array, but also in an $\frac{M}{b}$-gadget. The gadgets are independent random constructions (with different i.i.d. hash functions). The $O(\log n)$-bit key values are partitioned into distribution, shadow, and block hash fields when inserting and searching the gadgets in the nodes. When the capacity of the node fills, its keys are distributed to its children. Since $M \geq B^{1+\varepsilon}$ (the tall cache assumption), this distribution is I/O efficient, costing $O\left(\frac{1}{B}\right)$ per key.

The total cost for inserting a key is:

$$
O\left(\frac{1}{B} \log _{M} n\right)+U\left(\frac{M}{b}\right) \cdot \log _{M} n=O\left(\frac{\log _{M} n}{B}\right)+O\left(\log _{M} n\right) \cdot \frac{\lg M}{B \lg n} \cdot\left(\lg \lg M+t^{\min } \lg t^{\min }\right)
$$

Thus $t_{u}=O\left(\frac{1}{B}\right) \cdot\left(\log _{M} n+\lg \lg M+t^{\min } \lg t^{\min }\right)$. Note that $\log _{M} n+\lg \lg M=\Theta\left(\log _{M} n+\lg \lg n\right)$. For any $\lambda \geq \log _{M} n+\lg \lg n$, we can achieve $t_{u}=O(\lambda / B)$ by setting $t^{\min } \lg t^{\min }=\lambda$.

The cost of querying a key is $Q\left(\frac{M}{b}\right) \cdot O\left(\log _{M} n\right)=O\left(\frac{\lg M}{\lg t^{\min }} \cdot \log _{M} n\right)=O\left(\frac{\lg n}{\lg t^{\min }}\right)=O\left(\log _{\lambda} n\right)$.

The total space of our data structure is linear. Each key is stored in only one top-level gadget at a time, and, as we argued in 2.2.3 these are have linear size (in words).

\section{References}

[Arg03] Lars Arge. The buffer tree: A technique for designing batched external data structures. Algorithmica, 37(1):1-24, 2003. See also WADS'95.

[AV88] Alok Aggarwal and Jeffrey Scott Vitter. The input/output complexity of sorting and related problems. Communications of the ACM, 31(9):1116-1127, 1988. See also ICALP'87.

[BF03] Gerth S. Brodal and Rolf Fagerberg. Lower bounds for external memory dictionaries. In Proc. 14th ACM/SIAM Symposium on Discrete Algorithms (SODA), pages 546-554, 2003.

[Knu73] Donald E. Knuth. The Art of Computer Programming, Volume III: Sorting and Searching. Addison-Wesley, 1973. 
[PT07] Mihai Pătraşcu and Corina Tarniţă. On dynamic bit-probe complexity. Theoretical Computer Science, 380:127-142, 2007. See also ICALP'05.

[SSS95] Jeanette P. Schmidt, Alan Siegel, and Aravind Srinivasan. Chernoff-Hoeffding bounds for applications with limited independence. SIAM Journal on Discrete Mathematics, 8(2):223-250, 1995. See also SODA'93.

[VZ10] Elad Verbin and Qin Zhang. The limits of buffering: a tight lower bound for dynamic membership in the external memory model. In Proc. 41st ACM Symposium on Theory of Computing (STOC), pages $447-456,2010$.

[Yi10] Ke Yi. External memory data structures with o(1)-I/O updates. Dagstuhl Proceedings for "Data Structures" Meeting, 2010.

[YZ10] Ke Yi and Qin Zhang. On the cell probe complexity of dynamic membership. In Proc. 21st ACM/SIAM Symposium on Discrete Algorithms (SODA), pages 123-133, 2010.

\section{A Lower Bounds}

Formally, our model of computation is defined as follows. The update and query algorithms execute on a processor with $M$ bits of state, which is preserved from one operation to the next. The memory is an array of cells of $O(B \lg n)$ bits each, and allows random access in constant time. The processor is nonuniform: at each step, it decides on a memory cell as an arbitrary function of its state. It can either write the cell (with a value chosen as an arbitrary function of the state), or read the cell and update the state as an arbitrary function of the cell contents.

We first define our hard distribution. Choose $S$ to be a random set of $n$ keys from the universe $[2 n]$, and insert the keys of $S$ in random order. The sequence contains a single query, which appears at a random position in the second half of the stream of operations. In other words, we pick $t \in\left[\frac{n}{2}, n\right]$ and place the query after the $t$-th update (say, at time $t+\frac{1}{2}$ ).

Looking back in time from the query, we group the updates into epochs of $\lambda^{i}$ updates, where $\lambda$ is a parameter to be determined. Let $S_{i} \subset S$ be the keys inserted in epoch $i$; $\left|S_{i}\right|=\lambda^{i}$. Let $i_{\max }$ be the largest value such that $\sum_{i=1}^{i_{\max }} \lambda^{i} \leq \frac{n}{2}$ (remember that there are at least $n / 2$ updates before the query). We always construct $i_{\max }$ epochs. Let $i_{\min }$ be the smallest value such that $\lambda^{i_{\min }-1} \geq M$; remember that $M<n^{1-\varepsilon}$ was the cache size in bits. Our lower bound will generally ignore epochs below $i_{\min }$, since most of those elements may be present in the cache. Note $i_{\max }-i_{\min } \geq \varepsilon \log _{\lambda} n-O(1)$.

We have not yet specified the distribution of the query. Let $\mathcal{D}_{\mathrm{NO}}$ be the distribution in which the query is chosen uniformly at random from $[2 n] \backslash S$. Let $\mathcal{D}_{i}$ be the distribution in which the query is uniformly chosen among $S_{i}$. Then, $\mathcal{D}_{\text {YES }}=\frac{1}{i_{\max }-i_{\min }+1}\left(\mathcal{D}_{i_{\min }}+\cdots+\mathcal{D}_{i_{\max }}\right)$. Observe that elements in smaller epochs have a much higher probability of being the query. We will prove a lower bound on the mixture $\frac{1}{2}\left(\mathcal{D}_{\mathrm{YES}}+\mathcal{D}_{\mathrm{NO}}\right)$. Since we are working under a distribution, we may assume the data structure is deterministic by fixing its random coins (nonuniformly).

Observation 5. Assume the data structure has error $C_{\varepsilon}$ on $\frac{1}{2}\left(\mathcal{D}_{\mathrm{YES}}+\mathcal{D}_{\mathrm{NO}}\right)$. Then the error on $\mathcal{D}_{\mathrm{NO}}$ is at most $2 C_{\varepsilon}$. At least half the choices of $i \in\left\{i_{\min }, \ldots, i_{\max }\right\}$ are good in the sense that the error on $\mathcal{D}_{i}$ is at most $4 C_{\varepsilon}$. 
We will now present the high-level structure of our proof. We focus on some epoch $i$ and try to prove that a random query in $\mathcal{D}_{\mathrm{NO}}$ reads $\Omega(1)$ cells that are somehow "related" to epoch $i$. To achieve this, we consider the following encoding problem: for $k=\lambda^{i}$, the problem is to send a random bit vector $A[1 \ldots 2 k]$ with exactly $k$ ones. The entropy of $A$ is $\log _{2}\left(\begin{array}{c}2 k \\ k\end{array}\right)=2 k-O(\lg k)$ bits.

The encoder constructs a membership instance based on its array $A$ and public coins (which the decoder can also see). It then runs the data structure on this instance, and sends a message whose size depends on the efficiency of the data structure. Comparing the message size to the entropy lower bound, we get an average case cell-probe lower bound.

The encoder constructs a data structure instance as follows:

- Pick the position of the query by public coins. Also pick $S^{\star}=S \backslash S_{i}$ by public coins.

- Pick $Q=\left\{q_{1}, \ldots, q_{2 k}\right\}$ a random set of $k$ keys from $[2 n] \backslash S^{\star}$, also by public coins.

- Let $S_{i}=\left\{q_{j} \mid A[j]=1\right\}$, i.e. the message is encoded in the choice of $S_{i}$ out of $Q$.

- Run every query in $Q$ on the memory snapshot just before the position chosen in step 1.

The idea is to send a message, depending on the actions of the data structure, that will also allow the decoder to simulate the queries. Note that, up to the small probability of a query error, this allows the decoder to recover $A: A[j]=1$ iff $q_{j} \in S$.

It is crucial to note that the above process generates an $S$ that is uniform inside $[2 n]$. Furthermore, each $q_{j}$ with $A[j]=0$ is uniformly distributed over $[2 n] \backslash S$. That means that for each negative query, the data structure is being simulated on $\mathcal{D}_{\mathrm{NO}}$. For each positive query, the data structure is being simulated on $\mathcal{D}_{i}$.

Let $R_{i}$ be the cells read during epoch $i$, and $W_{i}$ be the cells written during epoch $i$ (these are random variables). We will use the convenient notation $W_{<i}=\bigcup_{j=0}^{i-1} W_{j}$.

The encoding algorithm will require different ideas for $t_{u}=1-\varepsilon$ and $t_{u}=o(1)$.

\section{A.1 Constant Update Time}

We now give a much simpler proof for the lower bound of [VZ10]: for any $t_{u} \leq 1-\varepsilon$, the query time is $t_{q}=\Omega\left(\log _{B} n\right)$.

We first note that the decoder can compute the snapshot of the memory before the beginning of epoch $i$, by simply simulating the data structure $\left(S_{>i}\right.$ was chosen by public coins). The message of the encoder will contain the cache right before the query, and the address and contents of $W_{<i}$. To bound $\left|W_{<i}\right|$ we use:

Observation 6. For any epoch $i, \mathbf{E}\left[\left|W_{i}\right|\right] \leq \lambda^{i} t_{u}$.

Proof. We have a sequence of $n$ updates, whose average expected cost (by amortization) is $t_{u}$ cell probes. Epochs $i$ is an interval of $\lambda^{i}$ updates. Since the query is placed randomly, this interval is shifted randomly, so its expected cost is $\lambda^{i} t_{u}$.

We start by defining a crucial notion: the footprint $\operatorname{Foot}_{i}(q)$ of query $q$, relative to epoch $i$. Informally, these are the cells that query $q$ would read if the decoder simulated it "to the best of its knowledge." Formally, we simulate $q$, but whenever it reads a cell from $W_{i} \backslash W_{<i}$, we feed into the simulation the value of the cell from before epoch $i$. Of course, the simulation may be incorrect if at least one cell from $W_{i} \backslash W_{<i}$ is read. However, we insist on a worst-case time of $t_{q}$ (remember that we allow Monte Carlo randomization, so we can put a worst-case bound on the query time). Let $\operatorname{Foot}_{i}(q)$ be the set of cells that the query algorithm reads in this, possibly bogus, simulation.

Note that the decoder can compute $\operatorname{Foot}_{i}(q)$ if it knows the cache contents at query time and $W_{<i}$ : it need only simulate $q$ and assume optimistically that no cell from $W_{i} \backslash W_{<i}$ is read. 
For some set $X$, let $\operatorname{Foot}_{i}(X)=\bigcup_{q \in X} \operatorname{Foot}_{i}(q)$. Now define the footprint of epoch $i: F_{i}=\left(\operatorname{Foot}_{i}\left(S_{i}\right) \cup\right.$ $\left.W_{i}\right) \backslash F_{<i}$. In other words, the footprint of an epoch contains the cells written in the epoch and the cells that the positive queries to that epoch would read in the decoder's optimistic simulation, but excludes the footprints of smaller epochs. Note that $F_{<i}=W_{<i} \cup\left(\bigcup_{j=0}^{i-1} \operatorname{Foot}_{j}\left(S_{j}\right)\right)$.

We are now ready for the main part of our proof. Let $H_{b}(\cdot)$ be the binary entropy.

Lemma 7. Let $i \in\left\{i_{\min }, \ldots, i_{\max }\right\}$ be good and $k=\lambda^{i}$. Let $p$ be the probability over $\mathcal{D}_{\mathrm{NO}}$ that the query reads a cell from $F_{i}$. We can encode a vector of $2 k$ bits with $k$ ones by a message of expected size: $O\left(\lambda^{i-1} t_{q} \cdot B \lg n\right)+2 k \cdot\left[H_{b}\left(\frac{1-\varepsilon}{2}\right)+H_{b}\left(\frac{p}{2}\right)+H_{b}\left(3 C_{\varepsilon}\right)\right]$.

Before we prove the lemma, we show it implies the desired lower bound. Choose $\lambda=B \lg ^{2} n$, which implies $\lambda^{i-1} t_{q} \cdot B \lg n=o\left(\lambda^{i}\right)=o(k)$. Note that $H_{b}\left(\frac{1-\varepsilon}{2}\right)$ is a constant bounded below 1 (depending on $\varepsilon$ ). On the other hand $H_{b}\left(3 C_{\varepsilon}\right)=O\left(C_{\varepsilon} \lg \frac{1}{C_{\varepsilon}}\right)$. Thus, there exist a small enough $C_{\varepsilon}$ depending on $\varepsilon$ such that $H_{b}(1-\varepsilon)+H_{b}\left(3 C_{\varepsilon}\right)<1$. Since the entropy of the message is $2 k-O(\lg k)$, we must have $H_{b}(1-\varepsilon)+H_{b}\left(3 C_{\varepsilon}\right)+H_{b}(p) \geq 1-o(1)$. Thus $H_{b}\left(\frac{p}{2}\right)=\Omega(1)$, so $p=\Omega(1)$.

We have shown that a query over $\mathcal{D}_{\mathrm{NO}}$ reads a cell from $F_{i}$ with constant probability, for any good $i$. Since half the $i$ 's are good and the $F_{i}$ 's are disjoint by construction, the expected query time must be $t_{q}=\Omega\left(\log _{\lambda} n\right)=\Omega\left(\log _{B} n\right)$.

Proof of Lemma 7 The encoding will consist of:

1. The cache contents right before the query.

2. The address and contents of cells in $F_{<i}$. In particular this includes $W_{<i}$, so the decoder can compute $\operatorname{Foot}_{i}(q)$ for any query.

3. Let $X \subseteq Q \backslash S_{i}$ be the set of negative queries that read at least one cell from $F_{i}$. We encode $X$ as a subset of $Q$, taking $\log _{2}\left(\begin{array}{c}2 k \\ |X|\end{array}\right)+O(\lg k)$ bits.

4. For each cell $c \in W_{i} \backslash F_{<i}$, mark some positive query $q \in S_{i}$ such that $c \in \operatorname{Foot}_{i}(q)$. Some cells may not mark any query (if they are not in the footprint of any positive query), and multiple cells may mark the same query. Let $M$ be the set of marked queries. We encode $M$ as a subset of $Q$, taking $\log _{2}\left(\begin{array}{c}2 k \\ |M|\end{array}\right) \leq \log _{2}\left(\begin{array}{c}2 k \\ \left|W_{i}\right|\end{array}\right)$ bits.

5. The set of queries of $Q$ that return incorrect results (Monte Carlo error).

The decoder immediately knows that queries from $X$ are negative, and queries from $M$ are positive. Now consider what happens if the decoder simulates some query $q \in Q \backslash(X \cup M)$. If $q$ reads some cell from $\operatorname{Foot}_{i}(M) \backslash F_{<i}$, we claim it must be a positive query. Indeed, $M \subset S_{i}$, so $\operatorname{Foot}_{i}(M) \backslash F_{<i} \subseteq F_{i}$. But any negative query that reads from $F_{i}$ was identified in $X$.

Claim 8. If a query $q \in Q \backslash(X \cup M)$ does not read any cell from $\operatorname{Foot}_{i}(M) \backslash F_{<i}$, the decoder can simulate it correctly.

Proof. We will prove that such a query does not read any cell from $W_{i} \backslash F_{<i}$, which means that the decoder knows all necessary cells. First consider positive $q$. If $q$ reads a cell from $W_{i} \backslash F_{<i}$ and $q$ is not marked, it means this cell marked some other $q^{\prime} \in M$. But that means the cell is in $\operatorname{Foot}_{i}\left(q^{\prime}\right) \subseteq \operatorname{Foot}_{i}(M)$, contradiction.

Now consider negative $q$. Note that $W_{i} \backslash F_{<i} \subseteq F_{i}$, so if $q$ had read a cell from this set, it would have been placed in the set $X$. 
Of course, some queries may give wrong answers when simulated correctly (Monte Carlo error). The decoder can fix these using component 5 of the encoding.

It remains to analyze the expected size of the encoding. To bound the binomial coefficients, we use the inequality: $\log _{2}\left(\begin{array}{c}n \\ m\end{array}\right) \leq n \cdot H_{b}\left(\frac{m}{n}\right)$. We will also use convexity of $H_{b}(\cdot)$ repeatedly.

1. The cache size is $M \leq \lambda^{i-1}$ bits, since $i \geq i_{\min }$.

2. We have $\mathbf{E}\left[\left|F_{<i}\right|\right] \leq \mathbf{E}\left[\left|W_{<i}\right|\right]+\left|S_{<i}\right| \cdot t_{q} \leq O\left(\lambda^{i-1} t_{q}\right)$. So this component takes $O\left(\lambda^{i-1} t_{q} \cdot B \lg n\right)$ bits on average.

3. Since $\mathbf{E}[|X|]=p k$, this takes $2 k \cdot H_{b}\left(\frac{p}{2}\right)$ bits on average.

4. Since $\mathbf{E}\left[\left|W_{i}\right|\right]=t_{u} k \leq(1-\varepsilon) k$, this takes $2 k \cdot H_{b}\left(\frac{1-\varepsilon}{2}\right)$ bits on average.

5. The probability of an error is at most $2 C_{\varepsilon}$ on $\mathcal{D}_{\mathrm{NO}}$ and $4 C_{\varepsilon}$ on $\mathcal{D}_{i}$. So we expect at most $6 C_{\varepsilon} \cdot k$ wrong answers. This component takes $2 k \cdot H_{b}\left(3 C_{\varepsilon}\right)$ bits on average.

This completes the proof of Lemma7 and our analysis of update time $t_{u}=1-\varepsilon$.

\section{A.2 Subconstant Update Time}

The main challenge in the constant regime was that we couldn't identify which cells were the ones in $W_{i}$; rather we could only identify the queries that read them. Since $t_{u} \ll 1$ here, we will be able to identify those cells among the cells read by queries $Q$, so the footprints are no longer a bottleneck.

The main bottleneck becomes writing $W_{<i}$ to the encoding. Following the trick of [PT07], our message will instead contain $W_{i} \cap R_{<i}$ and the cache contents at the end of epoch $i$. We note that this allows the decoder to recover $W_{<i}$. Indeed, the keys $S_{<i}$ are chosen by public coins, so the decoder can simulate the data structure after the end of epoch $i$. Whenever the update algorithm wants to read a cell, the decoder checks the message to see if the cell was written in epoch $i$ (whether it is in $W_{i} \cap R_{<i}$ ), and retrieves the contents if so.

We bound $W_{i} \cap R_{<i}$ by the following, which can be seen as a strengthening of Observation 6

Lemma 9. At least a quarter of the choices of $i \in\left\{i_{\min }, \ldots, i_{\max }\right\}$ are good and satisfy $\mathbf{E}\left[\left|W_{i} \cap R_{<i}\right|\right]=$ $O\left(\lambda^{i-1} t_{u} / \log _{\lambda} n\right)$.

Proof. We will now choose $i$ randomly, and calculate $\mathbf{E}\left[\left|W_{i} \cap R_{<i}\right| / \lambda^{i}\right]$, where the expectation is over the random distribution and random $i$. We will show $\mathbf{E}\left[\left|W_{i} \cap R_{<i}\right| / \lambda^{i}\right]=O\left(t_{u} /\left(\lambda \log _{\lambda} n\right)\right)$, from which the lemma follows by a Markov bound and union bound (ruling out the $i$ 's that are not good).

A cell is included in $W_{i} \cap R_{<i}$ if it is read at some time $r$ that falls in epochs $\{0, \ldots, i-1\}$, and the last time it was written is some $w$ that falls in epoch $i$. For fixed $w<r$, let us analyze the probability that this happens, over the random choice of the query's position. There are two necessary and sufficient conditions for the event to happen:

- the boundary between epochs $i$ and $i+1$ must occur before $w$, so the query must appear before $w+\sum_{j \leq i} \lambda^{j}<w+2 \lambda^{i}$. Since the query must also appear after $r$, the event is impossible unless $r-w<2 \lambda^{i}$.

- the boundary between epochs $i$ and $i-1$ must occur in $(w, r)$, so there are at most $r-w$ favorable choices for the query. Note also that the query must occur before $r+\sum_{j<i} \lambda^{j}$, so there are at most $2 \lambda^{i-1}$ favorable choices.

Let $i^{\star}$ be the smallest value such that $r-w<2 \lambda^{i^{\star}}$. Let us analyze the contribution of this operation to $\mathbf{E}\left[\left|W_{i} \cap R_{<i}\right| / \lambda^{i}\right]$ for various $i$. If $i<i^{\star}$, the contribution is zero. For $i=i^{\star}$, we use the bound $2 \lambda^{i-1}$ for 
the number of favorable choices. Thus, the contribution is $O\left(\frac{\lambda^{i-1}}{n} \cdot \lambda^{-i}\right)=O\left(\frac{1}{n \lambda}\right)$. For $i>i^{\star}$, we use the bound $r-w$ for the number of favorable choices. Thus, the contribution is $O\left(\frac{r-w}{n} \cdot \lambda^{-i}\right)=O\left(\frac{1}{n} \lambda^{i^{\star}-i}\right)$.

We conclude that the contribution is a geometric sum dominated by $O\left(\frac{1}{n \lambda}\right)$. Overall, there are at most $n \cdot t_{u}$ memory reads in expectation, so averaging over the choice of $i$, we obtain $\frac{1}{\log _{\lambda} n} \cdot O\left(\frac{1}{n \lambda}\right) \cdot n t_{u}=$ $O\left(\frac{t_{u}}{\lambda \log _{\lambda} n}\right)$.

Our main claim is:

Lemma 10. Let $i \in\left\{i_{\min }, \ldots, i_{\max }\right\}$ be chosen as in Lemma 9 and $k=\lambda^{i}$. Let $p$ be the probability over $\mathcal{D}_{\mathrm{NO}}$ that the query reads a cell from $W_{i} \backslash W_{<i}$. We can encode a vector of $2 k$ bits with $k$ ones by a message of expected size: $O\left(\lambda^{i-1} t_{u} \cdot B \lg \lambda\right)+O\left(k t_{u} \lg \frac{t_{q}}{t_{u}}\right)+2 k \cdot\left[H_{b}\left(\frac{p}{2}\right)+H_{b}\left(3 C_{\varepsilon}\right)\right]$.

We first show how the lemma implies the desired lower bound. Let $\lambda$ be such that the first term in the message size is $\leq \lambda^{i}=k$. This requires setting $\lambda=O\left(t_{u} B \lg \left(t_{u} B\right)\right)$.

Note that the lower bound is the same for $t_{u}=1-\varepsilon$ and, say, $t_{u}=1 / \sqrt{B}$. Thus, we may assume that $t_{u} \leq 1 / \sqrt{B} \leq 1 / \sqrt{\lg n}$. Therefore $k t_{u} \lg \frac{t_{q}}{t_{u}}=O\left(k \cdot \frac{\lg \lg n}{\sqrt{\lg n}}\right)=o(k)$.

Since the entropy is $2 k-o(\lg n)$, we must have $\frac{1}{2}+H_{b}\left(\frac{p}{2}\right)+H_{b}\left(3 C_{\varepsilon}\right) \geq 1-o(1)$. For a small enough $C_{\varepsilon}$, we obtain $H_{b}\left(\frac{p}{2}\right)=\Omega(1)$, so $p=\Omega(1)$.

We have shown that a query over $\mathcal{D}_{\mathrm{NO}}$ reads a cell from $W_{i} \backslash W_{<i}$ with constant probability, for at least a quarter of the choices of $i$. By linearity of expectation $t_{q}=\Omega\left(\log _{\lambda} n\right)=\Omega\left(\lg n / \lg \left(B t_{u}\right)\right)$.

Proof of Lemma 10, The encoding will contain:

1. The cache contents at the end of epoch $i$, and right before the query.

2. The address and contents of cells in $W_{i} \cap R_{<i}$. The decoder can recover $W_{<i}$ by simulating the updates after epoch $i$.

3. Let $X \subseteq Q \backslash S_{i}$ be the set of negative queries that read at least one cell from $W_{i} \backslash W_{<i}$. We encode $X$ as a subset of $Q$.

4. For each cell $c \in W_{i} \backslash W_{<i}$, mark some positive query $q \in S_{i}$ such that $c$ is the first cell from $W_{i} \backslash W_{<i}$ that $q$ reads. Note that distinct cells can only mark distinct queries, but some cells may not mark any query if no positive query reads them first among the set. Let $M$ be the set of marked queries, and encode $M$ as a subset of $Q$.

5. For each $q \in M$, encode the number of cell probes before the first probed cell from $W_{i} \backslash W_{<i}$, using $O\left(\lg t_{q}\right)$ bits.

6. The subset of queries from $Q$ that return a wrong answer (Monte Carlo error).

The decoder starts by simulating the queries $M$, and stops at the first cell from $W_{i} \backslash W_{<i}$ that they read (this is identified in part 5 of the encoding). The simulation cannot continue because the decoder doesn't know these cells. Let $W^{\star} \subseteq W_{i} \backslash W_{<i}$ be the cells where this simulation stops.

The decoder knows that queries from $M$ are positive and queries from $X$ are negative. It will simulate the other queries from $Q$. If a query tries to read a cell from $W^{\star}$, the simulation is stopped and the query is declared to be positive. Otherwise, the simulation is run to completion.

We claim this simulation is correct. If a query is negative and it is not in $X$, it cannot read anything from $W_{i} \backslash W_{<i}$, so the simulation only uses known cells. If a query is positive but it is not in $M$, it means either: (1) it doesn't read any cell from $W_{i} \backslash W_{<i}$ (so the simulation is correct); or (2) the first cell from $W_{i} \backslash W_{<i}$ that it reads is in $W^{\star}$, because it marked some other query in $M$. By looking at component 6 , the decoder can correct wrong answers from simulated queries.

It remain to analyze the size of the encoding: 
1. The cache size is $M=O\left(\lambda^{i-1}\right)$ bits.

2. By Lemma 9, $\mathbf{E}\left[\left|W_{i} \cap R_{<i}\right|\right]=O\left(\lambda^{i-1} \frac{t_{u}}{\log _{\lambda} n}\right)$, so this component takes $O\left(\lambda^{i-1} t_{u} \log \lambda \cdot B\right)$ bits.

3. Since $\mathbf{E}[|X|] \leq p \cdot k$, this component takes $\mathbf{E}\left[\log _{2}\left(\begin{array}{c}2 k \\ |X|\end{array}\right)\right] \leq 2 k \cdot H_{b}\left(\frac{p}{2}\right)$ bits.

4. Since $\mathbf{E}\left[\left|W_{i}\right|\right] \leq \lambda^{i} t_{u}=k t_{u}$, this takes $\mathbf{E}\left[\log _{2}\left(\begin{array}{c}2 k \\ \left|W_{i}\right|\end{array}\right)\right] \leq k \cdot O\left(t_{u} \lg \frac{1}{t_{u}}\right)$ bits.

5. This takes $\mathbf{E}\left[\left|W_{i}\right|\right] \cdot O\left(\lg t_{q}\right)=O\left(k t_{u} \lg t_{q}\right)$ bits.

6. We expect $2 C_{\varepsilon} \cdot k$ wrong negative queries and $4 C_{\varepsilon} \cdot k$ wrong positive queries, so this component takes $2 k \cdot H_{b}\left(3 C_{\varepsilon}\right)$ bits on average.

This completes the proof of Lemma 10, and our lower bound. 